SUBJECT AREAS:

MINERALOGY

SOLID-STATE CHEMISTRY

Received

31 July 2013

Accepted

13 September 2013

Published

2 October 2013

Correspondence and requests for materials should be addressed to N.I. (ishizawa@nitech. ac.jp)

\section{Structural evolution of calcite at high temperatures: Phase V unveiled}

\author{
Nobuo Ishizawa' ${ }^{1}$, Hayato Setoguchi' \& Kazumichi Yanagisawa ${ }^{2}$
}

${ }^{1}$ Advanced Ceramics Research Center, Nagoya Institute of Technology, 10-6-29 Asahigaoka, Tajimi, 507-0071, Japan, ${ }^{2}$ Research Laboratory of Hydrothermal Chemistry, Kochi University, 2-17-47 Asakurahoncho, Kochi, 780-8073, Japan.

The calcite form of calcium carbonate $\mathrm{CaCO}_{3}$ undergoes a reversible phase transition between $\mathrm{R} \overline{3} \mathrm{c}$ and $\mathrm{R} \overline{3} \mathrm{~m}$ at $\sim 1240 \mathrm{~K}$ under a $\mathrm{CO}_{2}$ atmosphere of $\sim 0.4 \mathrm{MPa}$. The joint probability density function obtained from the single-crystal X-ray diffraction data revealed that the oxygen triangles of the $\mathrm{CO}_{3}$ group in the high temperature form (Phase V) do not sit still at specified positions in the space group $\mathrm{R} 3 \mathrm{~m}$, but migrate along the undulated circular orbital about carbon. The present study also shows how the room temperature form (Phase I) develops into Phase V through an intermediate form (Phase IV) in the temperature range between $\sim 985 \mathrm{~K}$ and $\sim 1240 \mathrm{~K}$.

P receding Bragg's deduction in 1914 about the room-temperature atomic configuration of the calcite form of calcium carbonate $\mathrm{CaCO}_{3}$ (Phase I) ${ }^{1}$, Boeke found in 1912 a reversible phase transition at around $1243 \mathrm{~K}^{2}$. Since then, many experiments have been undertaken to determine the high-temperature structure of calcite and have indeed come very close to the final goal ${ }^{3}$, but none have succeeded in determining its true structure, for the following reason. In addition to the experimental difficulties associated with the instability of calcite tending towards decomposition into calcium oxide and carbon dioxide even under a $\mathrm{CO}_{2}$ atmosphere up to $\sim 0.4 \mathrm{MPa}$, the oxygen atoms do not sit at rest at the specific atom positions defined by the space group. Here we show the structure of the high temperature modification of calcite, named as Phase V by Mirwald ${ }^{4}$, in addition to the intermediate state (Phase IV) bridging Phases I and V. This study contributes, not only to crystallographic science, but also to a wider area including the carbon cycle of the earth, as the carbonate materials, being the abundant solid state container of carbon dioxide gas, are expected to follow a similar route of decarbonation as seen with calcite, irrelevant to temperature or atmosphere.

\section{Results}

The in-situ single-crystal X-ray diffraction experiments were carried out on calcite crystals at various temperatures up to $\sim 1300 \mathrm{~K}$ under a $\mathrm{CO}_{2}$ or air atmosphere (Tables S1-S4). The $h k 0$ and $h k 1$ reciprocal sections in the hexagonal setting at selected temperatures (Fig. 1) showed, in addition to the Bragg spots ascribed to the R $\overline{3} \mathrm{c}$ symmetry, diffuse scattering around reciprocal points with non-integer indices, which correspond to the ' $\mathrm{F}$ points' of the Brillouin zone of the $\mathrm{R} \overline{3} \mathrm{~m}$ parent lattice ${ }^{5}$. These diffuse $\mathrm{F}$ peaks became prominent upon heating. On the other hand, the $h k l$ reflections with $l=$ odd ('Z points' of the $\mathrm{R} \overline{3} \mathrm{~m}$ parent lattice) gradually weakened upon heating as first reported by Tsuboi in 1927 . The $\mathrm{Z}$ points barely existed at $1234 \mathrm{~K}$ and completely disappeared in the image taken at $1275 \mathrm{~K}$ (Fig. 1). This extinction provides the R $\overline{3} \mathrm{~m}$ symmetry for the crystal in Phase V. On the other hand, the diffuse F peaks still remained after the completion of transition into Phase $\mathrm{V}$, as evidenced in the same $1275 \mathrm{~K}$ image. This suggests that they do not originate from the $\mathrm{R} \overline{3} \mathrm{c}-\mathrm{R} \overline{3} \mathrm{~m}$ transition but underlie all the calcite variants. The transition temperature was estimated to be $1240 \mathrm{~K}$ from the linear extrapolation of the fourth power of the observed structure factor of the 113 reflection against temperature (Fig. S1c). Crystals in Phase V survived in a limited temperature range of $\sim 35 \mathrm{~K}$ between $1240-1275 \mathrm{~K}$, while precipitating a small amount of $\mathrm{CaO}$ powder under a $\mathrm{CO}_{2}$ atmosphere of $\sim 0.4 \mathrm{MPa}$ (Fig. S2a). The Phase I-IV-V transitions seemed reversible because the crystal cooled from $1275 \mathrm{~K}$ completely recovered the Phase I structure. Further heating above $1275 \mathrm{~K}$, on the other hand, caused the crystals a rapid decomposition into the $\mathrm{CaO}$ solid and $\mathrm{CO}_{2}$ gas without changing their apparent parallelepiped morphology (Fig. S2). Temperature dependencies of the cell dimensions were similar to those reported previously in detail ${ }^{7,8}(\mathrm{Fig}$. S1a-b).

The structure of Phase I consists of Ca1 at $(0,0,0), \mathrm{C} 1$ at $(0,0,1 / 4)$ and $\mathrm{O} 1$ at $(x, 0,1 / 4)$ with $x \sim 0.25$ in the space group $\mathrm{R} \overline{3} \mathrm{c}^{1,9-11}$ (Fig. 2a). All the carbonate groups on a plane parallel to (001) have the same orientation, whereas those in the adjacent planes have the inverse orientation with respect to the one in between. These 

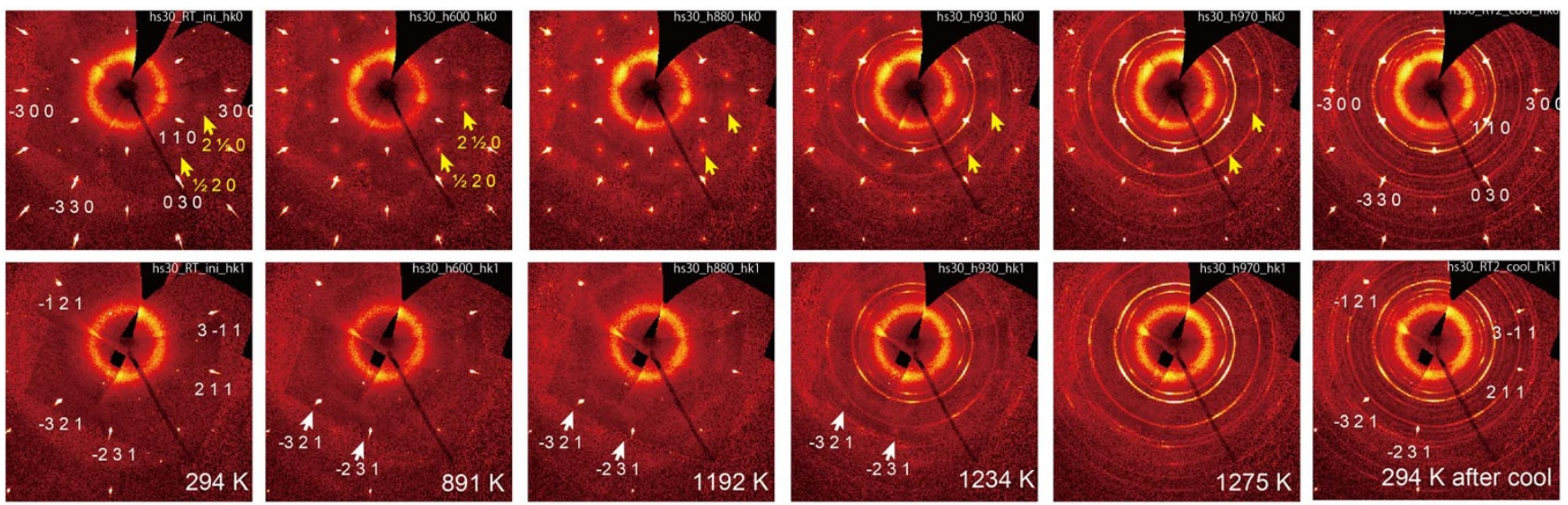

Figure $1 \mid$ Selected reciprocal sections of calcite upon heating and cooling. The $h k 0$ (top) and $h k 1$ (bottom) reciprocal sections of calcite (hs30) in the hexagonal setting, reconstructed from the two dimensional flame data at temperatures of 294 and $891 \mathrm{~K}$ (Phase I), 1192 and $1234 \mathrm{~K}$ (Phase IV) and $1275 \mathrm{~K}$ (Phase V) attained upon heating, and $294 \mathrm{~K}$ (Phase I) after cooling. Several Bragg spots are indexed based on the R $\overline{3} \mathrm{c}$ unit cell. Several diffuse ' $\mathrm{F}$ points are also indexed, for example, $21 / 20$, with yellow arrows.

different orientations are illustrated using different colours (blue and lime) for the $\mathrm{O} 1$ atoms. The structure of Phase IV also belongs to $\mathrm{R} \overline{3} \mathrm{c}$ but contains two different $\mathrm{O}$ atom sites in a disordered way at the Wyckoff notation $18 e$ : $\mathrm{O} 1(x, 0,1 / 4)$ and $\mathrm{O} 2(-x, 0,1 / 4)$ with $x \sim$ 0.25 . In Fig. 2 b, two different orientations of the $\mathrm{CO}_{3}$ groups are shown with the $\mathrm{O}$ atoms in blue and lime, in accordance with the colour scheme used in Fig. 2a. It should be noted that the two kinds of carbonate molecules in disorder are not in the $60^{\circ}$ or $180^{\circ}$ rotation relationships as often mentioned in literature, but in the improper relationship; the two molecules are geometrically related by the inversion at carbon, as evidenced by their atomic displacement parameter (ADP) ellipsoids (Fig. 2b and Fig. S3a).

The joint-probability density function was calculated from the inverse Fourier transform of the anharmonic ADPs approximated by the 3rd order expansion of the Gram-Charlier series ${ }^{12}$. The effective one particle potential was then obtained from the probability density function using Boltzmann statistics ${ }^{13}$. The potential profiles of the $\mathrm{O}$ triangle of the $\mathrm{CO}_{3}$ group (Fig. 3a-b) show thee deep minima at the $\mathrm{O} 1$ sites in Phase I, with a slight elongation along the $\mathrm{O}$ circle about carbon. On the other hand, the ridges separating the $\mathrm{O} 1$ sites become supressed upon heating in Phase IV, allowing existence of a small portion of $\mathrm{O}$ atoms at $\mathrm{O} 2$ sites. The potential barrier along the $\mathrm{O}$ circle changes with temperature (Fig. 3c), from which we could evaluate the activation energy as a function of temperature when the $\mathrm{O}$ atom hops between the $\mathrm{O} 1$ and $\mathrm{O}_{1}^{\prime}$ sites. The activation energy of approximately $1 \mathrm{eV}$ in Phase I is high enough to disable the migration of $\mathrm{O}$ atoms along the $\mathrm{O}$ circle. The single sharp ridge in Phase I becomes dented in Phase IV and splits into two gentle ridges between the $\mathrm{O} 1$ sites. These potential barriers become lower and shift towards the midpoint between $\mathrm{O} 1$ and $\mathrm{O} 2$ upon heating. The activation energy as a function of temperature (Fig. 3d) indicated a large drop of $\sim 0.5 \mathrm{eV}$ between $974 \mathrm{~K}$ and $995 \mathrm{~K}$, from which the IIV phase transition temperature was deduced to be $\sim 985 \mathrm{~K}$. The population of $\mathrm{O}$ atoms at $\mathrm{O} 2$ sites increased upon heating, with decreasing activation energy (Fig. 3d). The highest temperature of $1073 \mathrm{~K}$ that Markgraf and Reeder ${ }^{11}$ investigated is still at the a

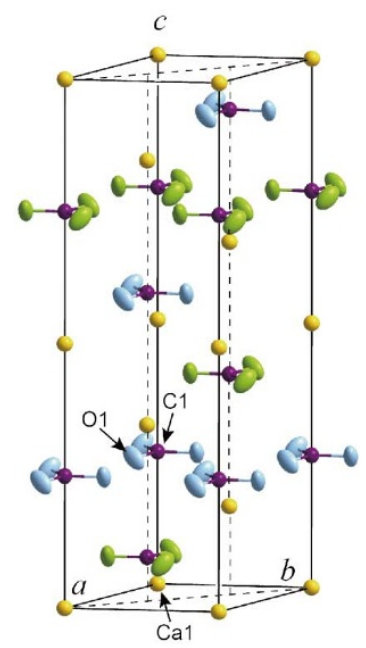

$787 \mathrm{~K}(\mathrm{I})$ b

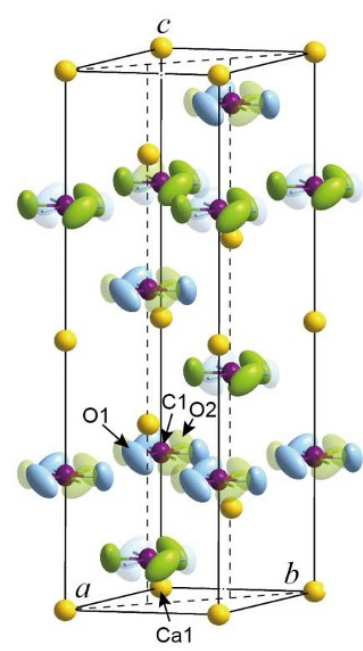

$1151 \mathrm{~K}(\mathrm{IV})$
C

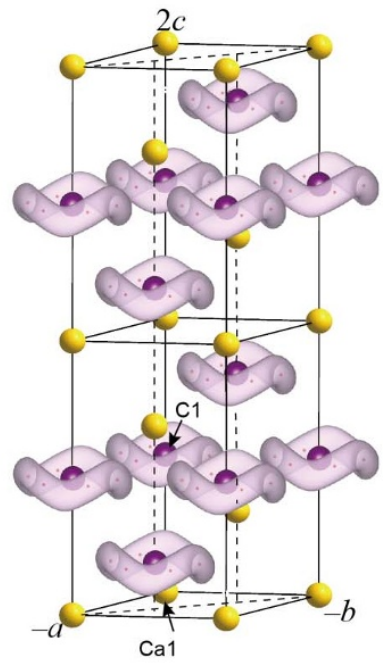

$1275 \mathrm{~K}(\mathrm{~V})$

Figure $2 \mid$ Structures of calcite. (a), Phase I at $787 \mathrm{~K},(\mathrm{~b})$, Phase IV at $1151 \mathrm{~K}$, (c), Phase V at $1275 \mathrm{~K}$. The ADP ellipsoids are drawn at the $50 \%$ probability level. The $\mathrm{O}$ atoms in Phase $\mathrm{V}$ are represented by the $50 \%$ probability isosurface of the joint probability density function. Two unit cells along (c) are drawn for Phase $V$ in the orientation, $a_{V}=-a_{I}$ and $b_{V}=-b_{I}$, so that Phase $V$ has an atom arrangement similar to those of Phases I and IV. This makes up for the change in the obverse-reverse relationships caused by the halving of the $\mathrm{R} \overline{\mathrm{c}} \mathrm{c}$ unit cell along $\mathrm{c}$. 
a

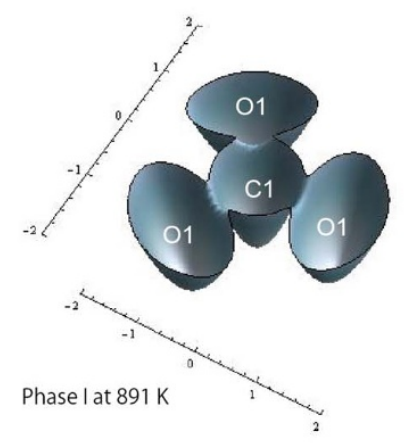

b

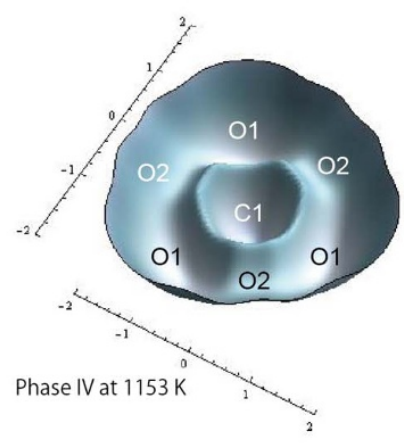

d

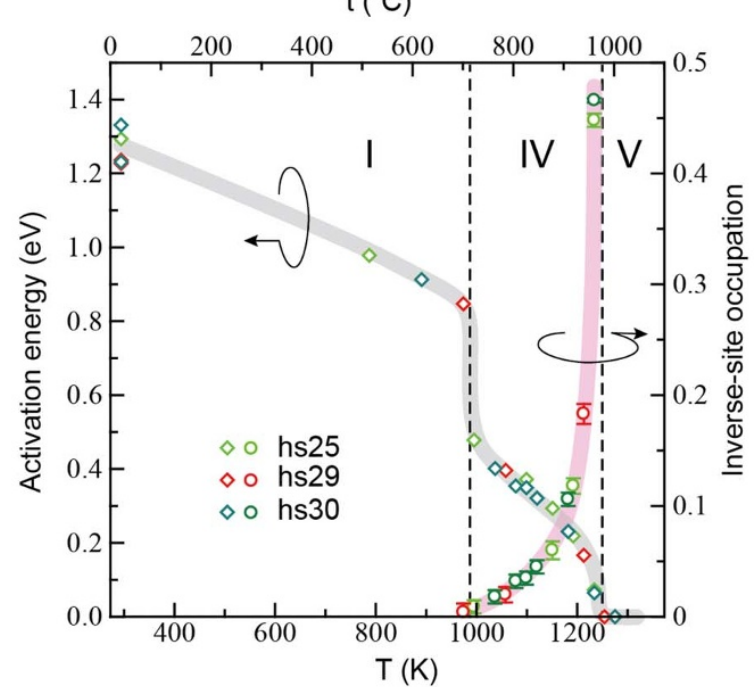

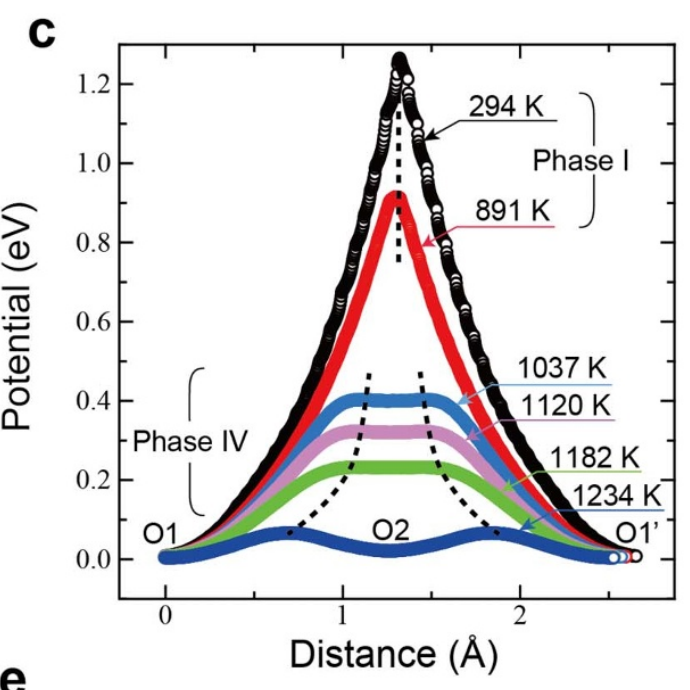

e

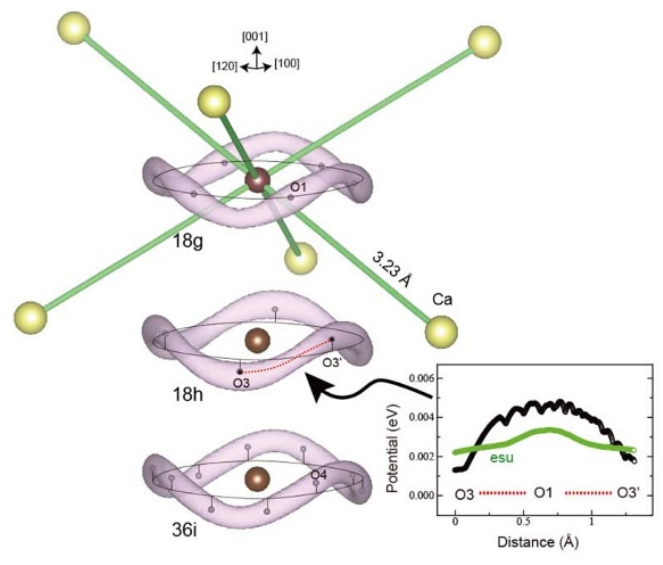

Figure 3 Potentials and the activation energy. (a), Potential surface of the $4 \AA \times 4 \AA$ square section (carbon at its centre) for Phase I at $891 \mathrm{~K}$ (hs 30 ). (b), That for Phase IV at $1153 \mathrm{~K}$ (hs30). The depth profiles in (a) and (b) are plotted in the range 0-0.7 eV. (c), Potential curves at selected temperatures (hs30) along the arc O1-O2-O1 in (b). Dashed lines indicate the expected traces of potential maxima in Phases I and IV. (d), The activation energy (eV) of the $\mathrm{O}$ atom along the $\mathrm{O}$ circle about carbon and the population of the inverse site $(\mathrm{O} 2)$ in Phase $\mathrm{IV}$ as a function of temperature. (e), The $10 \%$ isosurface of the joint probability density function of $\mathrm{O}$ atoms about carbon in the models $18 \mathrm{~g}, 18 \mathrm{~h}$ and $36 \mathrm{i}$ for Phase $\mathrm{V}$ at $1275 \mathrm{~K}$ (hs30). The six Ca atoms surrounding the $\mathrm{CO}_{3}$ group at $3.23 \AA$ are also drawn in the model $18 \mathrm{~g}$. The inset shows changes in potential energy (eV) along the dashed line in the undulated circular orbital with estimated standard uncertainties in green colour.

incipient period of Phase IV, where the $\mathrm{O} 2$ population is only $\sim 1 \%$. This is in agreement with a recent powder diffraction study ${ }^{8}$. Attainment of equal populations of $\mathrm{O} 1$ and $\mathrm{O} 2$ at the high temperature side of Phase IV means that the two moieties, $0 \leq z \leq 1 / 2$ and $1 /$ $2 \leq z \leq 1$ of the $\mathrm{R} \overline{3} \mathrm{c}$ unit cell become identical, resulting in a halved cell with the $\mathrm{R} \overline{3} \mathrm{~m}$ symmetry. This does not mean, however, that Phase $\mathrm{V}$ is a completely disordered version of Phase IV.

The difference Fourier synthesis with phases calculated from the $\mathrm{Ca}$ and $\mathrm{C}$ positions revealed residual electron densities distributed along an undulated circular orbital about carbon (Fig. S4b). This undulated orbital was modelled into three different arrangements of the $\mathrm{O}$ atoms in association with the use of anharmonic ADPs, as shown in Fig. 3e: 1) Model $18 \mathrm{~g}$ assuming half occupied $\mathrm{O} 1$ at Wyckoff position $18 g(x, 0,1 / 2)$ of the space group $\mathrm{R} \overline{3} \mathrm{~m}, 2)$ Model $18 \mathrm{~h}$ assuming half occupied $\mathrm{O} 3$ at $18 h(x,-x, z), 3)$ Model $36 \mathrm{i}$ assuming quarterly occupied $\mathrm{O} 4$ at the general position $36 i(x, y, z)$.

All these models gave similar reliability factors in the refinements. Moreover, they gave approximately the same joint probability density function regardless of the difference in the $\mathrm{O}$ atom positions. This indicates that the $\mathrm{O}$ atom does not sit still at a position specified by each model, but it can be located anywhere along the undulated circular orbital about carbon with equal probability. This 'itinerant' feature was also confirmed by the potential calculation along the undulated orbital (Fig. 3e inset). We saw in Phase IV that the $\mathrm{O}$ atom could migrate along the $\mathrm{O}$ circle by the hopping mechanism given the energy surpassing $0.2-0.4 \mathrm{eV}$. On the other hand, the potential curve in Phase $\mathrm{V}$ neither exceeds $\sim 0.005 \mathrm{eV}$ at any point, nor does it form a significant barrier exceeding three times of the estimated uncertainties. This means that the $\mathrm{O}$ atom can freely migrate along the undulated orbital about carbon without any energy for activation.

\section{Discussion}

The undulation of the $\mathrm{O}$ orbital presumably occurs in order to minimise the repulsion between the $\mathrm{Ca}$ and $\mathrm{O}$ electron clouds. As shown in Fig. 3e, the $\mathrm{O}$ orbital undulates as if avoiding the lines connecting $\mathrm{Ca}$ and $\mathrm{C}$. The shortest $\mathrm{Ca}-\mathrm{O}$ distances are 2.45, 2.38 and $2.37 \AA$ for the models $18 \mathrm{~g}, 18 \mathrm{~h}$ and $36 \mathrm{i}$, respectively. If no undulation is assumed, i.e., $z=1 / 2$ for $\mathrm{O}$ in the models $18 \mathrm{~h}$ and $36 \mathrm{i}$, the shortest $\mathrm{Ca}-\mathrm{O}$ distances become 2.27 and $2.26 \AA$, respectively, being $\sim 5 \%$ shorter than the undulated models and even shorter than the $\mathrm{Ca}-\mathrm{O}$ distance of $2.36 \AA$ in calcite at room temperature. Therefore, the undulation seemingly succeeds in realising reasonably long $\mathrm{Ca}-\mathrm{O}$ 

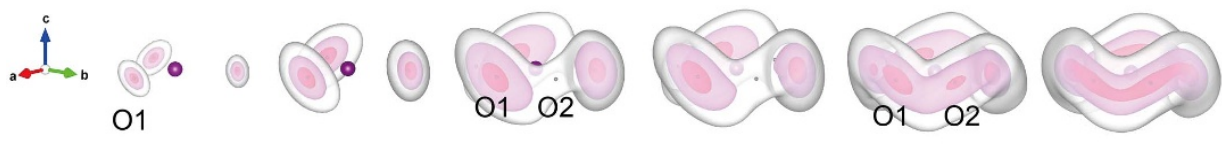

$294 \mathrm{~K}(\mathrm{I}) \quad 891 \mathrm{~K}(\mathrm{I}) \quad 1182 \mathrm{~K}(\mathrm{IV})$

$1213 \mathrm{~K}(\mathrm{IV})$

$1234 \mathrm{~K}(\mathrm{IV})$

$1275 \mathrm{~K}(\mathrm{~V})$

Figure 4 The isosurface plots of the joint probability density function of O atoms around carbon. Data plotted are at 294 and $891 \mathrm{~K}$ for Phase I, 1182 , 1213 , and $1234 \mathrm{~K}$ for Phase IV, and $1275 \mathrm{~K}$ for phase V. All data are calculated from the hs30 datasets, except for those at $1213 \mathrm{~K}$ from hs 29 . The isosurface levels are top $10 \%$ (red), 50\% (pink) and 90\% (white) probabilities from inside. The crystallographic (a) and (b) directions of the axes label are reversed in Phase $\mathrm{V}$ as explained in the caption of Fig. 2.

distances at any point of the orbital compared with the flat one. The undulation of the orbital along the $\mathrm{c}$ axis at $1275 \mathrm{~K}$ is $\pm 0.18(1) \AA$ at the maximum, which makes the inclination angles of $\pm 7.7(1)^{\circ}$ for the $\mathrm{C}-\mathrm{O}$ bond with respect to the basal plane. This sort of umbrella inversion between $+7.7^{\circ}$ and $-7.7^{\circ}$ (Fig. S3b) associated with the assumed rotational migration would drive the crystal to decarbonation shortly.

Finally, we stress the importance of the joint probability density function rather than the conventional ball-and-stick model in order to distinguish the Phases I, IV and V (Fig. 4). The three O1 atoms around carbon exist in an isolated manner in Phase I. The tail of the joint probability density function gradually extends to cover the $\mathrm{O} 2$ site in Phase IV. Although a portion of the $\mathrm{O}$ atoms can actually occupy the $\mathrm{O} 2$ site orienting to the inverse direction against the $\mathrm{O} 1$ triangle in Phase IV, the $\mathrm{O} 1$ and $\mathrm{O} 2$ atoms are still vibrating about their mean positions on the plane where carbon lies. Upon further heating, the joint probability density function is completely connected to form the undulated orbital in Phase V. The $\mathrm{O}$ atom sublattice is premelted and the positions become unable to be allocated specifically to any Wyckoff sites with fixed coordinates. The $\mathrm{O}$ atoms exist just as a probability along the undulated orbital about carbon. The present study has thus unveiled the Phase V structure of calcite, which stands as a crystal at the very last minute before decomposition.

\section{Methods}

Crystal growth. Reagent grade $\mathrm{CaCO}_{3}$ powder $(5.00 \mathrm{~g})$ was placed in a $1.4 \mathrm{~L}$ Teflonlined stainless steel autoclave with $1050 \mathrm{~mL}$ of $5 \mathrm{M} \mathrm{NH}_{4} \mathrm{NO}_{3}$ at $\mathrm{pH}$ 7.5. The autoclave was heated to $418 \mathrm{~K}$ for $7 \mathrm{~h}$ with agitation of a rotator at $250 \mathrm{rpm}$ to dissolve the calcite powder completely, followed by cooling at a rate of $2.5 \mathrm{~K} / \mathrm{h}$ to room temperature. The products were then washed with deionised distilled water and filtered using a $150 \mu \mathrm{m}$ sieve. Spectroscopic analysis using a JED-2300 instrument (Jeol Ltd.) revealed no significant impurities in the calcite crystals.

Diffraction experiments. Single-crystal X-ray diffraction experiments were carried out on several calcite crystals with a parallelepiped shape of $\sim 200 \mu \mathrm{m}$ edge length on average using an APEX II diffractometer (Bruker AXS Inc.) equipped with a chargecoupled device X-ray detector and a high temperature apparatus ${ }^{14}$. Shield tube Mo K $\alpha$ was used with the $0.3 \varphi$ monocap capillary collimator. The hs11 datasets were collected in an open air atmosphere up to $787 \mathrm{~K}$ using a crystal mounted on a silica glass capillary with the adhesive cement. The hs 25,29 and 30 datasets were collected using crystals which were each encapsulated in a thin $0.2-0.3 \varphi$ silica glass capillary under the $\mathrm{CO}_{2}$ atmosphere. No adhesive was necessary in this case because the crystal was always kept in tight contact with the inner wall of capillary at elevated temperatures and rarely slipped, owing to the difference in thermal expansion between the crystal and the silica glass (Fig. S2a). The $\mathrm{CO}_{2}$ pressure in the sealed capillary was estimated to be $\sim 0.4 \mathrm{MPa}$ at $\sim 1300 \mathrm{~K}$ from the pressure-temperature law for the ideal gas. The crystal in capillary was placed in a hot air stream, the temperature of which was monitored by the $\mathrm{Pt} / \mathrm{Pt} 13 \% \mathrm{Rh}$ thermocouple. The sample temperature was calibrated using the $3 \mathrm{rd}$ order polynomial according to our previous study ${ }^{15}$. The accuracy of the transition temperature $1240 \mathrm{~K}$ was estimated to be approximately $\pm 5 \mathrm{~K}$. A set of frame data at a constant temperature was collected for $3-5$ h up to $2 \theta<60^{\circ}$ for the hs 11 and hs 25 datasets, $2 \theta<80^{\circ}$ for hs 29 and $2 \theta<70^{\circ}$ for hs 30 .

Data analysis. Crystallographic calculations were primarily conducted using Jana2006 ${ }^{16}$. Vesta ${ }^{17}$, Diamond (Crystal Impact GbR) and Mathematica (Wolfram Research, Inc) were used for graphical representations and other subsidiary calculations. Extinction corrections were applied on the basis of the Becker \& Coppens ${ }^{18}$ formalism (Type I) to the Phase I datasets. The extinction effect was negligibly small for the Phase IV and V datasets. In parallel with the anharmonic ADP refinements, the translation-libration-screw (TLS) refinements ${ }^{12}$, using a symmetric 3 $\times 3$ translation tensor $\mathrm{T}$, the symmetric $3 \times 3$ libration tensor $\mathrm{L}$ and the nonsymmetric $3 \times 3$ screw tensor $S$, were carried out assuming a rigid body model for the $\mathrm{CO}_{3}$ group with the 321 point symmetry. Further details are given in Supplementary information.

1. Bragg, W. L. The analysis of crystals by the X-ray spectrometer. Proceedings of the Royal Society of London. Series A 89, 468-489 (1914).

2. Boeke, H. E. Die Schmelzerscheinungen und die umkehrbare Umwandlung des Calciumcarbonats. Neues Jahrb. Mineral. 1, 91-121 (1912).

3. Dove, M. T., Swainson, I. P., Powell, B. M. \& Tennant, D. C. Neutron powder diffraction study of the orientational order-disorder phase transition in calcite, $\mathrm{CaCO}_{3}$. Phys. Chem. Minerals 32, 493-503 (2005).

4. Mirwald, P. W. A differential thermal analysis study of the high-temperature polymorphism of calcite at high pressure. Contr. Mineral. and Petrol. 59, 33-40 (1976).

5. Hagen, M., Dove, M. T., Harris, M. J., Steigenberger, U. \& Powell, B. M. Orientational order-disorder phase transition in calcite. Physica B: Condensed Matter 180-181, 276-278 (1992).

6. Tsuboi, C. On the Effect of temperature upon the crystal Structure of calcite. Proceedings of the Imperial Academy (Japan) 3, 17-18 (1927).

7. Dove, M. T. \& Powell, B. M. Neutron diffraction study of the tricritical orientational order/disorder phase transition in calcite at $1260 \mathrm{~K}$. Phys. Chem. Minerals 16, 503-507 (1989).

8. Antao, S. M., Hassan, I., Mulder, W. H., Lee, P. L. \& Toby, B. H. In situ study of the R-3c to R-3m orientational disorder in calcite. Phys. Chem. Minerals 36, 159-169 (2009).

9. Wyckoff, R. W. G. The crystal structures of some carbonates of the calcite group. American Journal of Science, Series 4 50, 317-360 (1920).

10. Maslen, E. N., Streltsov, V. A. \& Streltsova, N. R. X-ray study of the electron density in calcite, $\mathrm{CaCO}_{3}$. Acta Crystallogr., Sect. B: Struct. Sci. 49, 636-641 (1993).

11. Markgraf, S. A. \& Reeder, R. J. High-temperature structure refinements of calcite and magnesite. Amer. Mineral. 70, 590-600 (1985).

12. Willis, B. T. M. \& Pryor, A. W. Thermal vibrations in crystallography. (Cambridge University Press, 1975).

13. Zucker, U. H. \& Schulz, H. Statistical approaches for the treatment of anharmonic motion in crystals. I. A comparison of the most frequently used formalisms of anharmonic thermal vibrations. Acta Crystallogr., Sect. A: Found. Crystallogr. 38, 563-568 (1982).

14. Ishizawa, N. et al. Development of high-temperature single-crystal X-ray diffraction system for electron density distribution analyses. Annu. Rep. CRL 2006, Nagoya Inst. Technol. 6, 12-18 (2007).

15. Wang, J. W., Hibino, H. \& Ishizawa, N. Temperature calibration of the hightemperature single-crystal X-ray dffractometer. Annu. Rep. CRL 2010, Nagoya Inst. Technol. 10, 43-47 (2011).

16. Petricek, V., Dusek, M. \& Palatinus, L. Jana2006, Structure Determination Software Programs (Institute of Physics, Praha, Czech Republic, 2006).

17. Momma, K. \& Izumi, F. VESTA 3 for three-dimensional visualization of crystal, volumetric and morphology data. J. Appl. Crystallogr. 44, 1272-1276 (2011).

18. Becker, P. J. \& Coppens, P. Extinction within the limit of validity of the Darwin transfer equations. I. General formalism for primary and secondary extinction and their applications to spherical crystals. Acta Crystallogr., Sect. A: Found. Crystallogr. 30, 129-147 (1974).

\section{Acknowledgements}

We thank Dr Vaclav Petricek at the Institute of Physics, Academy of Science, Czech Republic, for his invaluable support with our data calculation and implementation of the Jana2006 program package, Ms. Tomomi Gotoda at Research Laboratory of Hydrothermal Chemistry, Kochi University, for her help with the growth of calcite crystals, Mr. Hisashi Hibino at Nagoya Institute of Technology, for his help with the EDS and SEM analyses, Associate Professor Jung Wang from the Inner Mongolia University of Technology, and Dr Terutoshi Sakakura from the Institute of Multidisciplinary Research for Advanced Materials, Tohoku University, Japan, for their help in the preliminary stage of this work. This work was supported by JSPS KAKENHI Grant number 22360272.

\section{Author contributions}

K.Y. conducted the hydrothermal growth of calcite crystals. H.S. conducted all the diffraction experiments at high temperatures and EDS/SEM experiments. N.I. carried out the remaining parts of the study. 


\section{Additional information}

Supplementary information accompanies this paper at http://www.nature.com/ scientificreports

Competing financial interests: The authors declare no competing financial interests.

How to cite this article: Ishizawa, N., Setoguchi, H. \& Yanagisawa, K. Structural evolution of calcite at high temperatures: Phase V unveiled. Sci. Rep. 3, 2832; DOI:10.1038/srep02832 (2013).

(c) (1) (2) This work is licensed under a Creative Commons AttributionBY SA NonCommercial-ShareAlike 3.0 Unported license. To view a copy of this license, visit http://creativecommons.org/licenses/by-nc-sa/3.0 